Intrinsic deviations in fluorescence yield detected x-ray absorption spectroscopy: the case of the transition metal $\mathrm{L}_{2,3}$ edges

This article has been downloaded from IOPscience. Please scroll down to see the full text article.

2012 J. Phys.: Condens. Matter 24452201

(http://iopscience.iop.org/0953-8984/24/45/452201)

View the table of contents for this issue, or go to the journal homepage for more

Download details:

IP Address: 131.211.213.168

The article was downloaded on 01/02/2013 at 14:38

Please note that terms and conditions apply. 


\title{
Intrinsic deviations in fluorescence yield detected $x$-ray absorption spectroscopy: the case of the transition metal $L_{2,3}$ edges
}

\author{
Reshmi Kurian $^{1}$, Kristjan Kunnus ${ }^{2}$, Philippe Wernet $^{2}$, \\ Sergei M Butorin ${ }^{3}$, Pieter Glatzel ${ }^{4}$ and Frank M F de Groot ${ }^{1}$ \\ ${ }^{1}$ Inorganic Chemistry and Catalysis, Debye Institute for Nanomaterials Science, Utrecht University, \\ Universiteitsweg 99, 3584 CG, The Netherlands \\ ${ }^{2}$ Institute for Methods and Instrumentation for Synchrotron Radiation Research, Helmholtz-Zentrum \\ Berlin für Materialien und Energie GmbH, Albert-Einstein-Straße 15, D-12489 Berlin, Germany \\ ${ }^{3}$ Department of Physics and Astronomy, Uppsala University, Box 516, SE-751 20 Uppsala, Sweden \\ ${ }^{4}$ European Synchrotron Radiation Facility, BP 220, F-38043 Grenoble Cedex 9, France
}

E-mail: R.Kurian@uu.nl and F.M.F.deGroot@uu.nl

Received 21 June 2012, in final form 25 September 2012

Published 12 October 2012

Online at stacks.iop.org/JPhysCM/24/452201

\begin{abstract}
Fluorescence yield (FY) detected x-ray absorption spectra (XAS) of 3d transition metal ions are calculated from the integrated $2 \mathrm{p} 3 \mathrm{~d}$ resonant $\mathrm{X}$-ray emission spectra. The resulting FY-XAS spectra are compared with the normal XAS spectra corresponding to the absorption cross section and significant deviations between the two spectra are found. This implies that the assumption that the FY-XAS spectrum identifies with the XAS spectrum is disproved. Especially for the early transition metal systems the differences between the FY-XAS and XAS are large, due to the opening of inelastic decay channels from selected X-ray absorption final states. The theoretical calculations show that the difference between FY detection and XAS is largest for the detection in depolarized geometry. The calculations are compared with experimental spectra for oxides and coordination compounds for $\mathrm{Fe}^{2+}, \mathrm{Co}^{2+}$ and $\mathrm{Ni}^{2+}$ systems. The implications for the sum rules in XAS and magnetic circular dichroism experiments are discussed.
\end{abstract}

(Some figures may appear in colour only in the online journal)

\section{Introduction}

The 2p x-ray absorption spectra ( $\mathrm{L}_{2,3}$ edges) of $3 \mathrm{~d}$ transition metal systems are generally measured with electron yield (EY) and fluorescence yield (FY) detection [1]. Transmission measurements are less often applied because the strong absorption cross sections imply the need for a uniform sample thickness below of the order of $1 \mu \mathrm{m}[2,3]$. X-ray microscopy experiments regularly make use of the transmission mode [4, 5] and since the first application of transmission mode XAS to water by Yang and Kirz in 1985 [6] approaches have been developed recently for transmission mode detected XAS of water and aqueous solutions [7-13]. Still, most 2p XAS experiments are performed with EY measurements, that use drain current, total electron yield detectors (i.e. channeltrons) or the integrated photoemission spectrum. EY measurements can suffer from surface induced effects, they need vacuum or low-pressure conditions, they can be affected by electric and magnetic fields [14] and they can suffer from saturation [15]. The alternative detection scheme is with FY. Self-absorption effects in FY yield detection may result in strongly distorted spectral intensities. Dilute systems can ideally be measured 
with FY and concentrated systems can sometimes be diluted and/or corrected for saturation effects [1].

It has been shown that the FY spectra differ from the $\mathrm{X}$-ray absorption cross sections or 'true XAS spectra' not only due to saturation effects and self-absorption, but also due to the intrinsic processes of the FY decay. In 1994 it was shown that the FY detected spectrum of a nickel-cyanide complex does not relate to the $2 p$ XAS spectrum due to the state dependent intensity fluctuations. The $2 p$ XAS spectrum can be described as a series of excitations to localized $2 \mathrm{p}^{5} 3 \mathrm{~d}^{N+1}\{\Gamma\}$ final states, each with a different specific symmetry $\Gamma$. It turned out to be the case that each different final state $\Gamma$ has its own specific decay strengths for Auger channels and for radiative channels. Calculations showed that the variation in the Auger channels was only $20 \%$, mainly due to the relative importance of state independent core-core-core Auger. The radiative decay is dominated by the $2 \mathrm{p} 3 \mathrm{~d}$ XES channel that showed variations of $500 \%$ over the $2 p$ XAS spectrum [16]. Because the states with high radiative decay are concentrated more on the high energy side of the $\mathrm{L}_{3}$ and the $\mathrm{L}_{2}$ edges, this state dependent decay also implies a strong energy dependence. The same mechanism is responsible for the difference between FY-XAS and XAS at the $\mathrm{M}_{5}$ edges of rare earth systems [17]. In contrast, most experimental studies involving experimental FY spectra do not seem to be affected by distortions from the XAS spectral shape, or at least they are not analyzed for the possibility of being distorted. To shed some more light on this situation, we have carried out a systematic investigation on transition metal spectra for typical systems ranging from $3 \mathrm{~d}^{1}$ to $3 \mathrm{~d}^{9}$ ionic ground states.

We use a theoretical approach according to which the FY signal is dominated by the $2 \mathrm{p} 3 \mathrm{~d}$ resonant $\mathrm{x}$-ray emission spectroscopy (RXES) channels, and calculate the integrated 2p3d RXES spectral shape. Effectively, this can be approximated as the convolution of $2 \mathrm{p}$ XAS and $2 \mathrm{p} 3 \mathrm{~d}$ XES, slightly modified for interference effects. We show that FY detected XAS spectra contain intrinsic deviations from the $\mathrm{X}$-ray absorption cross sections in the case of the L edges of $3 \mathrm{~d}$ transition metal systems. The reason for these deviations is found to be the state dependence of the fluorescence channels.

A core hole decays via fluorescence decay and via Auger decay. We define the state dependent decay constants as respectively $k_{\mathrm{FY}}(\Gamma)$ and $k_{\mathrm{AUG}}(\Gamma)$. Each $2 \mathrm{p}$ core hole state $\Gamma$ has its specific energy, which implies that the state dependent decay also gives an energy dependent decay. O'Brien et al showed that for localized core level excitonic states in insulators, the valence-band emission is lowered in energy due to the screening by the core exciton [18]. The exact energy dependence is modified by the respective lifetime broadenings and interference effects. Under the assumptions (i) that a core hole decays via both fluorescence yield $k_{\mathrm{FY}}(\Gamma)$ and Auger decay $k_{\mathrm{AUG}}(\Gamma)$ and (ii) that the decay channels are independent, the decay rate $(\mathrm{d} / \mathrm{d} t)$ for the total number of core holes $(N)$ is given as

$$
\frac{\mathrm{d} N(\Gamma, t)}{\mathrm{d} t}=-N(\Gamma, t)\left(k_{\mathrm{FY}}(\Gamma)+k_{\mathrm{AUG}}(\Gamma)\right) .
$$

This defines the intensity of the fluorescence decay $\left(I_{\mathrm{FY}}(\Gamma)\right)$ in terms of the decay constants as the normalized fraction of the decay constants

$$
I_{\mathrm{FY}}(\Gamma) \propto \frac{k_{\mathrm{FY}}(\Gamma)}{k_{\mathrm{AUG}}(\Gamma)+k_{\mathrm{FY}}(\Gamma)} .
$$

In the case of the $\mathrm{L}$ edges of $3 \mathrm{~d}$ transition metals, the core hole decays mainly via Auger decay with $k_{\mathrm{AUG}}(\Gamma)$ being 100 times larger than $k_{\mathrm{FY}}(\Gamma)$. In addition, the combined Auger channels can be considered as constant, as has been shown in detail for $\mathrm{Ni}^{2+}[16]$. With these two approximations the fluorescence decay can be written as

$$
I_{\mathrm{FY}}(\Gamma) \propto \frac{k_{\mathrm{FY}}(\Gamma)}{k_{\mathrm{AUG}}(\Gamma)+k_{\mathrm{FY}}(\Gamma)} \cong \frac{k_{\mathrm{FY}}(\Gamma)}{k_{\mathrm{AUG}}} .
$$

Note that if the core hole were to decay mainly via fluorescence yield and all fluorescence was detected in the experiment, this equation would yield an approximately state independent constant fluorescence yield intensity.

The fluorescence yield decay constant is in this work calculated as the integral over the $2 \mathrm{p} 3 \mathrm{~d}$ x-ray emission channel, which we will show to be strongly state dependent. In principle, this is not the only dipole allowed XES decay channel and alternative decay channels are 2p3s XES and $2 \mathrm{p} 4 \mathrm{~s}$ XES. Calculations of the radial matrix elements confirm that the 2p3s XES decay channel is weaker by at least a factor of four for all systems. The $2 p 4 s$ decay channel is much weaker still due to a small matrix element and few occupied $4 \mathrm{~s}$ (character) states. Because 2p3s XES involves the replacement of a $2 p$ core hole with a 3 s core hole, this decay is only dependent on the number of $2 p$ core holes in each intermediate state, which is exactly 1.0 for all $2 \mathrm{p}$ XAS states. This implies that the $2 \mathrm{p} 3 \mathrm{~s}$ XES is state independent and does not show an energy dependence. The constant $2 \mathrm{p} 3 \mathrm{~s}$ XES implies that the combined effect of $2 \mathrm{p} 3 \mathrm{~d}$ and $2 \mathrm{p} 3 \mathrm{~s}$ XES decay is a combination of state independent 2p3s XES and state dependent $2 \mathrm{p} 3 \mathrm{~d}$ XES. Note that because the 2p3s XES decay does not show an energy dependence it can be used as a PFY detector channel to reveal directly the XAS spectral shape.

There are two effects that could affect the calculated FY-XAS results: (1) super Coster-Kronig (SCK) Auger decay channels and (2) charge transfer effects. SCK Auger involves a decay channel from the $L_{2}$ edge to the $L_{3}$ with the emission of a $\sim 10 \mathrm{eV}$ electron. This is a relatively strong channel. The $\mathrm{SCK} \mathrm{L}_{3}$ state will subsequently decay via other (cascade) Auger channels, but a small fraction will decay via the radiative $2 \mathrm{p} 3 \mathrm{~d}$ XES channel, adding intensity to the FY-XAS signal. Recent experiments that detect complete RIXS planes of $\mathrm{Cr}^{3+}$ systems confirm the presence of these SCK Auger plus 2p3d XES cascade channels [19]. The SCK Auger decay has two counterbalancing effects on the FY-XAS intensity: (i) the shortened lifetime of the $\mathrm{L}_{2}$ states decreases the FY-XAS of the $\mathrm{L}_{2}$ edge and (ii) the SCK Auger decay followed by $2 \mathrm{p} 3 \mathrm{~d}$ XES cascade channels increases the FY-XAS of the $\mathrm{L}_{2}$ edge. In the present calculations we do not include charge transfer effects. Charge transfer states have a different degree of localization and it can be expected that the ratio between radiative and non-radiative channels is modified. This implies that systems with significant charge 
transfer intensity, in particular involving both ligand-metal and metal-ligand charge transfer in molecular $\pi$-systems [20, 21 ], can show modified results. On the other hand, we do not expect significant modifications in the case of bulk oxides.

In section 3 of this paper we systematically study

(a) the deviation in the FY-XAS spectra from the XAS spectra for the $3 \mathrm{~d}^{1-9}$ transition metal ions;

(b) polarization dependent effects on the FY-XAS spectra;

(c) the XAS versus FY-XAS for the low-spin $3 d^{4}, 3 d^{5}, 3 d^{6}$ and $3 \mathrm{~d}^{7}$ systems;

(d) the consequence for the sum rules of the XAS and FY-XAS spectral intensities;

(e) the effect of the inclusion of coherence between the $2 p$ XAS and 2p3d XES transitions;

(f) comparison with experimental spectra for $3 d^{6}, 3 d^{7}$ and $3 \mathrm{~d}^{8}$ systems.

\section{Experiment and theory}

\subsection{Computational details}

We have applied crystal field multiplet theory to calculate the $2 p$ XAS ( $\mathrm{L}_{2,3}$ edge) spectra and 2p3d RXES spectral shapes. The $2 p$ XAS spectra have been calculated as the dipole transitions from $3 \mathrm{~d}^{N}$ to $2 \mathrm{p}^{5} 3 \mathrm{~d}^{N+1}$ configurations. The simulations are carried out with the crystal field multiplet approach developed by Thole et al $[22,23]$. The atomic values of the Slater integrals are used, which we calculate by scaling the Hartree-Fock calculated values by 0.8 . Because we intend to use a simple model that explains the main features of FY detection, charge transfer effects have been neglected. The $2 \mathrm{p} 3 \mathrm{~d}$ RXES calculations involve the transition from the $3 \mathrm{~d}^{N}$ ground state to the $3 \mathrm{~d}^{N}$ final states through the $2 \mathrm{p}^{5} 3 \mathrm{~d}^{N+1}$ intermediate states. This transition is theoretically described by the Kramers-Heisenberg $(\mathrm{KH})$ formula $[14,24,25]$ and the corresponding matrices involved are the $3 \mathrm{~d}^{N}$ to $2 \mathrm{p}^{5} 3 \mathrm{~d}^{N+1}$ dipole transition matrices coupled to the (identical) $2 \mathrm{p}^{5} 3 \mathrm{~d}^{N+1}$ to $3 \mathrm{~d}^{N}$ dipole decay matrices. The scattering intensity $F$ as a function of incident $(\Omega)$ and emitted x-ray energy $(\omega)$ is given as

$$
\begin{aligned}
& F(\Omega, \omega)= \\
& \sum_{j} \sum_{q, q^{\prime}}\left|\sum_{i} \frac{\left\langle 3 \mathrm{~d}^{N}\left|T_{2}\right| 2 \mathrm{p}^{5} 3 \mathrm{~d}^{N+1}\right\rangle\left\langle 2 \mathrm{p}^{5} 3 \mathrm{~d}^{N+1}\left|T_{1}\right| 3 \mathrm{~d}^{N}\left[\Psi_{0}\right]\right\rangle}{E_{3 \mathrm{~d}^{N}}+\Omega-E_{2 \mathrm{p}^{5} 3 \mathrm{~d}^{N+1}}+\mathrm{i} \Gamma_{2 \mathrm{p}^{5} 3 \mathrm{~d}^{N+1}}}\right|^{2} \\
& \times \delta(\Delta E+\Omega-\omega)
\end{aligned}
$$

where $j$ and $i$ indicate the $3 \mathrm{~d}^{N}$ final state and $2 \mathrm{p}^{5} 3 \mathrm{~d}^{N+1}$ intermediate state respectively. The $3 \mathrm{~d}^{N}$ ground state, $2 \mathrm{p}^{5} 3 \mathrm{~d}^{N+1}$ intermediate state and $3 \mathrm{~d}^{N}$ final state energies $(E)$ are given in the equation. $\Omega$ and $\omega$ are the energies of the incident and emitted photon, respectively, and $\Gamma$ indicates the lifetime broadening of the intermediate state. $T_{1}$ and $T_{2}$ indicate the dipole operators for absorption and emission. All calculations have been performed in $C_{4}$ symmetry, yielding nine polarization combinations of the left, right and $z$-polarised $\mathrm{x}$-rays. Using a correlation function description, a full RXES calculation involves potentially different polarizations for the absorption and emission step, implying $3^{4}=81$ polarization combinations [26]. We have chosen to assume a fixed polarization for the absorption and also for the emission step, which limits the 81 combinations to the nine combinations that we have treated. This is indicated in the $\mathrm{KH}$ equation as a summation over the polarizations $q$ and $q^{\prime}$. The transition lines have been convoluted using Lorentzian and Gaussian functions to account for the core hole life time and instrumental resolution respectively. Because the FY signal is integrated over the final states, the lifetime broadening of the final states and the experimental resolution of the x-ray detector do not influence the FY spectrum. The half width at half maximum of the Lorentzian used is $0.2 \mathrm{eV}$ for the $2 \mathrm{p}^{5} 3 \mathrm{~d}^{N+1}$ intermediate state and $0.2 \mathrm{eV}$ for the $3 \mathrm{~d}^{N}$ final state. The intermediate state lifetime broadening of $0.2 \mathrm{eV}$ is the average value of a typical $\mathrm{L}_{3}$ edge of a transition metal oxide. The final state lifetime broadening is less well known and could be lower than the value of $0.2 \mathrm{eV}$ that we use. We use a Gaussian broadening of $0.2 \mathrm{eV}$ to simulate a $200 \mathrm{meV}$ resolution monochromator, realizing that many actual beamlines have better resolution.

\subsection{Experiment}

The data for $\mathrm{LiFePO}_{4}[27,28]$ and $\mathrm{Co}_{2} \mathrm{SiO}_{4}$ [28] were measured at beamline I-511 [29], MAX-lab, Lund, Sweden. The total electron yield spectra were recorded in drain-current mode at $90^{\circ}$ radiation incidence angle to the surface of the samples while the total fluorescence yield was detected with the help of a multichannel plate detector at $45^{\circ}$ outgoing photon angle (in the horizontal plane). The samples were in powder form on conducting carbon tape. The experimental data on $\mathrm{Cs}\left[\mathrm{NiCr}(\mathrm{CN})_{6}\right] \cdot 2 \mathrm{H}_{2} \mathrm{O}$ have been digitized from [16].

\section{Results and discussion}

\subsection{The XAS and FY-XAS of transition metal ions}

The comparison between the XAS and FY-XAS for the $2 p$ XAS spectra of the transition metals is presented in figure 1 . The spectra have been generated as the combination of all nine polarization combinations. Figure 2 shows the effects in the case of the most used experimental polarizations. The influence of the $3 \mathrm{~d}$ spin-orbit coupling on the absorption spectra and the FY-XAS is given in the same figure, where the lower (upper) panel shows the result with (without) $3 \mathrm{~d}$ spin-orbit coupling. The maximum intensities of XAS and FY-XAS are normalized to unity. The transition metal $2 p$ levels split into $2 \mathrm{p}_{1 / 2}$ and $2 \mathrm{p}_{3 / 2}$ due to the spin-orbit coupling. Therefore, the energy separation between the $\mathrm{L}_{3}$ and $\mathrm{L}_{2}$ edges is mainly related to the spin-orbit coupling on the core $2 p$ levels of the transition metal ion. Table 1 details the Hund's rule ground state as well as the high-spin and low-spin orbital configurations of all the considered $3 \mathrm{~d}^{N}$ states. Below we analyze the specific issues of each $3 \mathrm{~d}^{N}$ ground state.

The $3 \mathrm{~d}^{1}$ system $\left(\mathrm{Ti}^{3+}\right)$ is shown in figure $1(\mathrm{a})$. The $3 \mathrm{~d}$ spin-orbit coupling modifies the details of the spectral shape. 

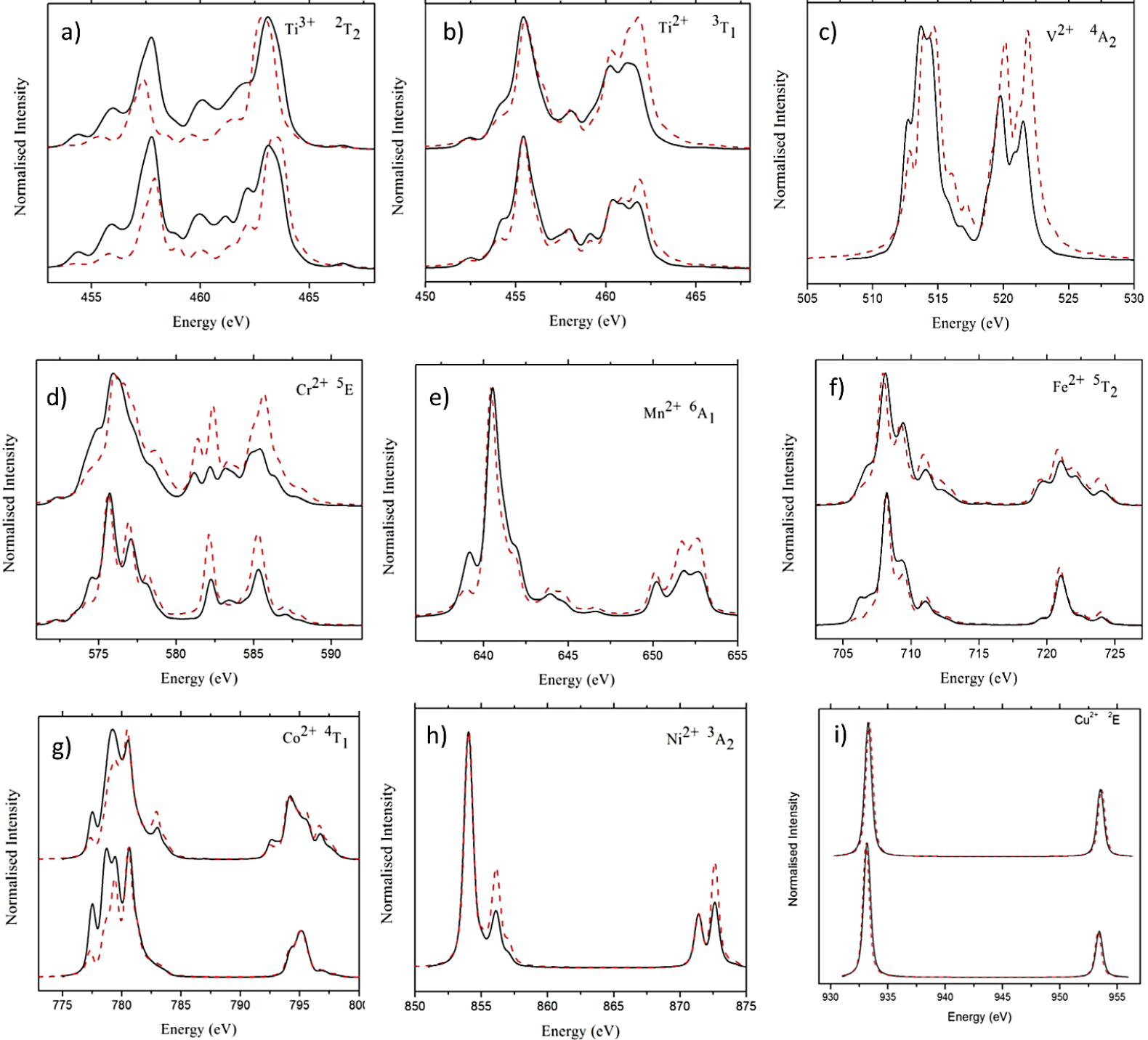

Figure 1. XAS and FY-XAS for the $3 \mathrm{~d}^{1}-3 \mathrm{~d}^{9}$ transition metal ions with a $10 \mathrm{Dq}$ value of $1.2 \mathrm{eV}$. The lower panel shows the results with $3 \mathrm{~d}$ spin-orbit coupling and the upper panel without $3 \mathrm{~d}$ spin-orbit coupling. The black solid line shows the XAS and the red dotted line is the FY-XAS spectrum.

Table 1. The calculations were performed for the ions as indicated in the first column. The second column indicates the atomic Hund's rule ground state. Columns 3 and 4 give the high-spin ground state for an octahedral $\left(\mathrm{O}_{\mathrm{h}}\right)$ crystal field. The one-electron orbital occupations are only approximate (typically $80-95 \%$ pure) due to $3 \mathrm{~d} 3 \mathrm{~d}$ interactions. Columns 5 and 6 give the high-spin ground state for an octahedral $\left(\mathrm{O}_{\mathrm{h}}\right)$ crystal field. If the ground state symmetry has $T_{1}, T_{2}$ or $E$ symmetry it can be affected by the $3 \mathrm{~d}$ spin-orbit coupling indicated by $+\mathrm{LS}$.

\begin{tabular}{llllll}
\hline Configuration & $\begin{array}{l}\text { Atomic } \\
\text { ground state }\end{array}$ & $\begin{array}{l}\text { High-spin } \\
\text { occupation } \\
\text { (approximate) }\end{array}$ & $\begin{array}{l}\text { High-spin ground } \\
\text { state in } \mathrm{O}_{\mathrm{h}}\end{array}$ & $\begin{array}{l}\text { Low-spin occupation } \\
\text { (approximate) }\end{array}$ & $\begin{array}{l}\text { Low-spin ground } \\
\text { state in } \mathrm{O}_{\mathrm{h}}\end{array}$ \\
\hline $3 \mathrm{~d}^{1}\left(\mathrm{Ti}^{3+}\right)$ & ${ }^{2} \mathrm{D}_{3 / 2}$ & $\mathrm{t}_{2 \mathrm{~g}+}^{1}$ & ${ }^{2} T_{2+\mathrm{LS}}$ & - & - \\
$3 \mathrm{~d}^{2}\left(\mathrm{Ti}^{2+}\right)$ & ${ }^{3} \mathrm{~F}_{2}$ & $\mathrm{t}_{2 \mathrm{~g}+}^{2}$ & ${ }^{3} T_{1+\mathrm{LS}}$ & - & - \\
$3 \mathrm{~d}^{3}\left(\mathrm{~V}^{2+}\right)$ & ${ }^{4} \mathrm{~F}_{3 / 2}$ & $\mathrm{t}_{2 \mathrm{~g}+}^{3}$ & ${ }^{4} A_{2}$ & - & - \\
$3 \mathrm{~d}^{4}\left(\mathrm{Cr}^{2+}\right)$ & ${ }^{5} \mathrm{D}_{0}$ & $\mathrm{t}_{2 \mathrm{~g}+}^{3} \mathrm{e}_{\mathrm{g}+}^{1}$ & ${ }^{5} E_{+\mathrm{LS}}$ & $\mathrm{t}_{2 \mathrm{~g}+}^{3} \mathrm{t}_{2 \mathrm{~g}-}^{1}$ & ${ }^{3} T_{1+\mathrm{LS}}$ \\
$3 \mathrm{~d}^{5}\left(\mathrm{Mn}^{2+}\right)$ & ${ }^{6} \mathrm{~S}_{5 / 2}$ & $\mathrm{t}_{2 \mathrm{~g}+}^{3} \mathrm{e}_{\mathrm{g}+}^{2}$ & ${ }^{6} A_{1}$ & $\mathrm{t}_{2 \mathrm{~g}+}^{3} \mathrm{t}_{2 \mathrm{~g}-}^{2}$ & ${ }^{2} T_{2+\mathrm{LS}}$ \\
$3 \mathrm{~d}^{6}\left(\mathrm{Fe}^{2+}\right)$ & ${ }^{5} \mathrm{D}_{2}$ & $\mathrm{t}_{2 \mathrm{~g}+}^{3} \mathrm{e}_{\mathrm{g}+}^{2} \mathrm{t}_{2 \mathrm{~g}-}^{1}$ & ${ }^{5} T_{2+\mathrm{LS}}$ & $\mathrm{t}_{2 \mathrm{~g}+}^{3} \mathrm{t}_{2 \mathrm{~g}-}^{3}$ & ${ }^{1} A_{1}$ \\
$3 \mathrm{~d}^{7}\left(\mathrm{Co}^{2+}\right)$ & ${ }^{4} \mathrm{~F}_{9 / 2}$ & $\mathrm{t}_{2 \mathrm{~g}+}^{3} \mathrm{e}_{\mathrm{g}+}^{2} \mathrm{t}_{2 \mathrm{~g}-}^{2}$ & ${ }^{4} T_{1+\mathrm{LS}}$ & $\mathrm{t}_{2 \mathrm{~g}+}^{3} \mathrm{e}_{\mathrm{g}+}^{1} \mathrm{t}_{2 \mathrm{~g}-}^{3}$ & ${ }^{2} E_{+\mathrm{LS}}$ \\
$3 \mathrm{~d}^{8}\left(\mathrm{Ni}^{2+}\right)$ & ${ }^{3} \mathrm{~F}_{4}$ & $\mathrm{t}_{2 \mathrm{~g}+}^{3} \mathrm{e}_{\mathrm{g}+}^{2} \mathrm{t}_{2 \mathrm{~g}-}^{3}$ & ${ }^{3} A_{2}$ & - & - \\
$3 \mathrm{~d}^{9}\left(\mathrm{Cu}^{2+}\right)$ & ${ }^{2} \mathrm{D}_{5 / 2}$ & $\mathrm{t}_{2 \mathrm{~g}+}^{3} \mathrm{e}_{\mathrm{g}+}^{2} \mathrm{t}_{2 \mathrm{~g}-}^{3} \mathrm{e}_{\mathrm{g}-}^{1}$ & ${ }^{2} E_{+\mathrm{LS}}$ & - & - \\
\hline
\end{tabular}



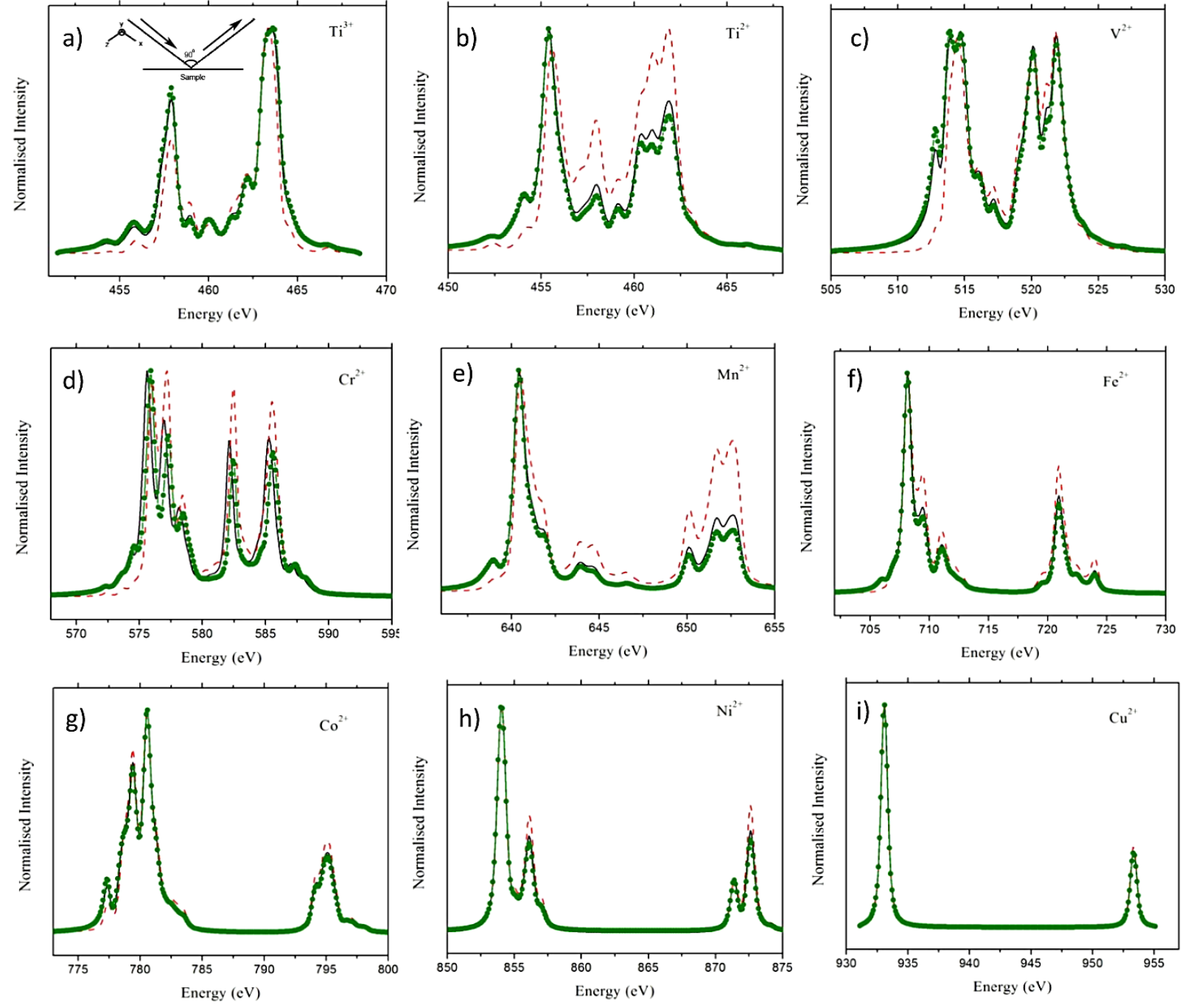

Figure 2. The polarization dependence of the FY-XAS for the $3 \mathrm{~d}^{1}-3 \mathrm{~d}^{9}$ transition metals is given. The spectra given are FY-XAS (black solid), FY-XAS (LH) (red dashed) and FY-XAS (LV) (green with symbol). The inset in (a) schematically shows the polarized and depolarized geometries used in the calculations (see text for details).

A discrepancy in the $\mathrm{L}_{3}-\mathrm{L}_{2}$ intensity ratios for the XAS versus FY-XAS is seen for both the calculations. The $3 \mathrm{~d}^{2}$ systems $\left(\mathrm{Ti}^{2+}\right)$ are shown in figure $1(\mathrm{~b})$. Similarly to $\mathrm{Ti}^{3+}$, different peaks in between the $\mathrm{L}_{3}$ and $\mathrm{L}_{2}$ edges are seen with $3 \mathrm{~d}$ spin-orbit coupling. A clear effect is that the $\mathrm{L}_{2}$ edge gains more intensity in the FY-XAS spectrum compared to XAS.

The $3 \mathrm{~d}^{3}$ systems $\left(\mathrm{V}^{2+}\right)$ are shown in figure $1(\mathrm{c})$. It is clear from figure 1(c) that the FY-XAS intensity of the $\mathrm{L}_{2}$ edge drastically increases and the $\mathrm{L}_{2}$ edge intensity becomes close to the intensity of the $\mathrm{L}_{3}$ edge. The $3 \mathrm{~d}^{4}$ systems $\left(\mathrm{Cr}^{2+}\right)$ are given in figure 1(d). The intensity of the high energy part of the $\mathrm{L}_{3}$ edge is slightly elevated for the FY-XAS spectra with/without inclusion of $3 \mathrm{~d}$ spin-orbit coupling. Moreover, there is a clear increase of the $\mathrm{L}_{2}$ edge in the FY-XAS spectrum in both cases. Spectra for the low-spin ground state are shown in figure 3(a). Similarly to the high-spin case, a strong increase in the $\mathrm{L}_{2}$ edge intensity is seen for the FY-XAS spectra compared to the XAS.

The $3 \mathrm{~d}^{5}$ systems $\left(\mathrm{Mn}^{2+}\right)$ are given in figure $1(\mathrm{e})$. This $A_{1}$ symmetry state is, in first approximation, not affected by the
$3 \mathrm{~d}$ spin-orbit coupling, but due to the interplay with mixing of excited symmetry states a small splitting can occur also for $\mathrm{Mn}^{2+}$ systems. Similarly to $\mathrm{Cr}^{2+}$ a slight elevation at the high energy part of the $\mathrm{L}_{3}$ edge and a clear increase of the $\mathrm{L}_{2}$ edge in the FY-XAS spectrum are visible. The low-spin $3 \mathrm{~d}^{5}$ systems are shown in figure 3(b) which shows that the trend of the FY-XAS spectrum compared to XAS is similar to the trend of the high-spin state. The $3 \mathrm{~d}^{6}$ systems $\left(\mathrm{Fe}^{2+}\right)$ are given in figure 1(f). The general trend of the FY-XAS spectrum in comparison with XAS is a slight decrease of the low energy part of the $\mathrm{L}_{3}$ edge and a slight increase of the high energy parts of the $L_{3}$ and $L_{2}$ edges. The low-spin $3 d^{6}$ systems are shown in figure 3(c). A slight increase of the high energy parts of the $\mathrm{L}_{3}$ and $\mathrm{L}_{2}$ edges is evident for the FY-XAS spectrum. The $3 \mathrm{~d}^{7}$ systems $\left(\mathrm{Co}^{2+}\right)$ are given in figure $1(\mathrm{~g})$. The intensity trend is similar to the high-spin $\mathrm{Fe}^{2+}$ system. The low-spin $3 \mathrm{~d}^{7}$ systems are shown in figure $3(\mathrm{~d})$. There is a decrease of the FY-XAS spectrum at the low energy part of the $\mathrm{L}_{3}$ edge and throughout the $\mathrm{L}_{2}$ edge. 

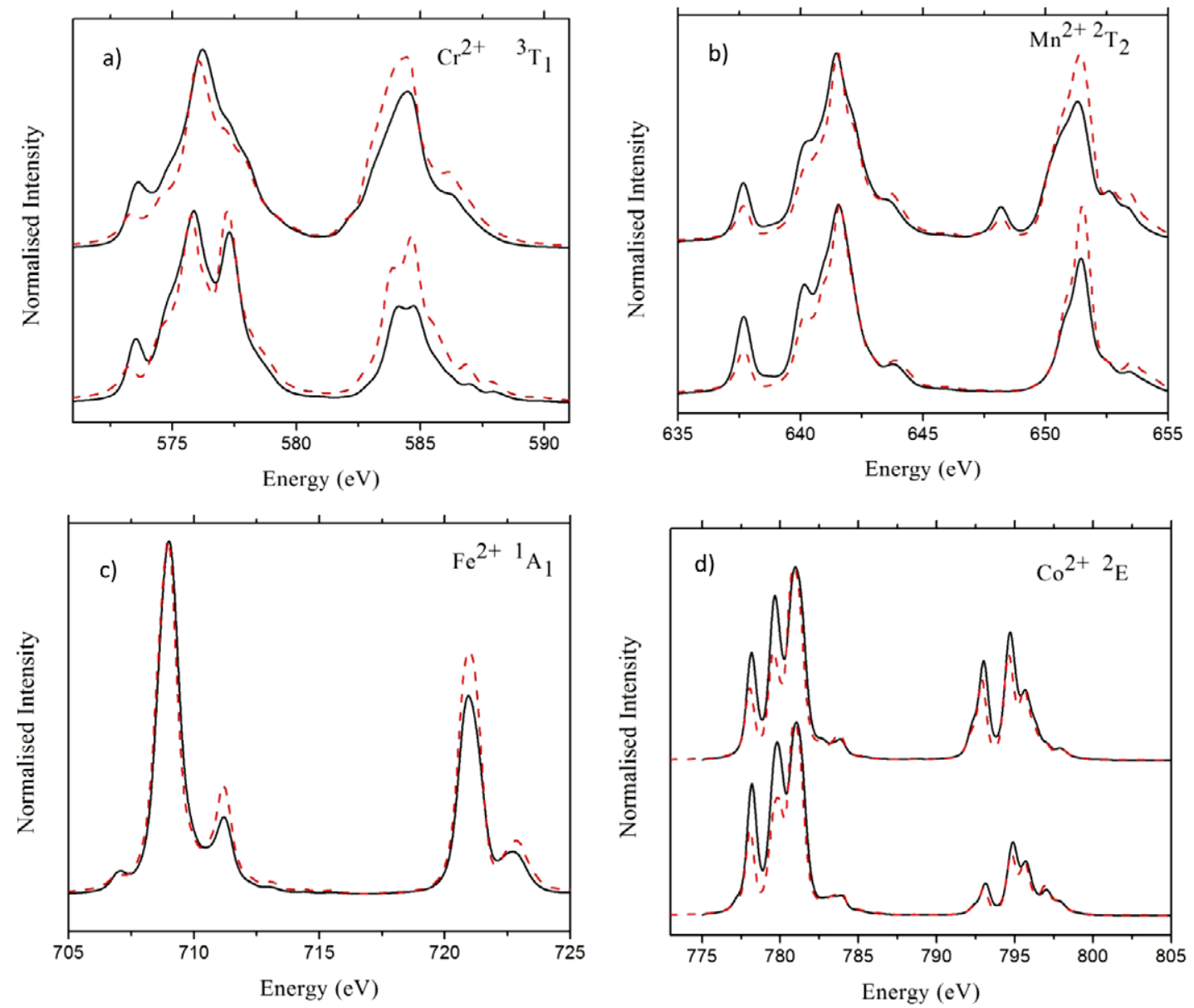

Figure 3. The low-spin XAS and FY-XAS spectra for the $3 \mathrm{~d}^{4}-\mathrm{d}^{7}$ transition metal ions. The lower panel shows the results with $3 \mathrm{~d}$ spin-orbit coupling and the upper panel without $3 \mathrm{~d}$ spin-orbit coupling. The ${ }^{1} A_{1}$ ground state of low-spin $\mathrm{Fe}^{2+}$ is not affected by $3 \mathrm{~d}$ spin-orbit coupling. The black solid line shows the XAS and the red dotted line the FY-XAS spectra.

$3 \mathrm{~d}^{8}$ systems $\left(\mathrm{Ni}^{2+}\right)$ are given in figure $1(\mathrm{~h})$. The intensity of the FY-XAS spectrum increases at the high energy parts of the $\mathrm{L}_{3}$ and $\mathrm{L}_{2}$ edges. The $3 \mathrm{~d}^{9}$ systems $\left(\mathrm{Cu}^{2+}\right)$ are given in figure $1(\mathrm{j})$. Without the $3 \mathrm{~d}$ spin-orbit coupling, the $\mathrm{L}_{3}-\mathrm{L}_{2}$ edge intensity ratio is 2:1 (cf figure $1(\mathrm{j})$ ). With $3 \mathrm{~d}$ spin-orbit coupling the branching ratio, defined as the intensity of the $\mathrm{L}_{3}$ edge divided by the total intensity of the $2 p$ XAS spectrum, is affected. In fact, the branching ratio is a direct measure of the mixing of the $3 \mathrm{~d}_{3 / 2}$ character into the $3 \mathrm{~d}_{5 / 2}$ atomic ground state due to crystal field effects.

Comparing all nine calculations in figure 1, one can make the general observations that the FY-XAS spectra have an increased intensity of the $\mathrm{L}_{2}$ edge and in addition the states at the higher ends of both the $\mathrm{L}_{3}$ and $\mathrm{L}_{2}$ edges increase in intensity. The states at higher energy on average seem to have more and stronger fluorescent decay channels. The increase of the $\mathrm{L}_{2}$ edge also implies that the branching ratio is significantly affected in FY-XAS spectra.

\subsection{Polarization dependent effects}

In photon-in photon-out experiments, the polarization and scattering angle are important additional degrees of freedom.
The angular dependence of the integrated RIXS and RXES as well as the form of the sum rules are studied and reported by Ferriani et al [30-33]. Depending on the polarization of the $\mathrm{x}$-rays used as well as the scattering angle, different combinations of polarizations of the $\mathrm{x}$-ray absorption and $x$-ray emission transitions are combined. The results for the $3 \mathrm{~d}$ transition metals for the high-spin states are given in figure 2. In figure 2, we compare the total FY-XAS spectrum with the two most commonly applied experimental settings for soft $\mathrm{x}$-ray emission experiments, where the angle between the incoming $\mathrm{x}$-ray propagation direction and the outgoing $\mathrm{x}$-rays is set to $90^{\circ}$; the setup is schematically shown in the inset of figure 2(a). The incoming $x$-rays are polarized either linear parallel (depolarized geometry or linear horizontal; LH) or linear perpendicular (polarized geometry or linear vertical; LV) to the scattering plane. It is interesting to note that the LV polarized spectra are essentially identical to the total polarization averaged spectra. The LH polarized spectra show differences with the LV spectra and the polarization averaged spectra. In general the LH spectra have an increased difference with the XAS spectra, as compared with the polarization averaged FY-XAS spectra: the peaks of the $\mathrm{L}_{2}$ edge and the peaks at the high energy side of the $\mathrm{L}_{3}$ and $\mathrm{L}_{2}$ edges are further enhanced in LH polarization. 


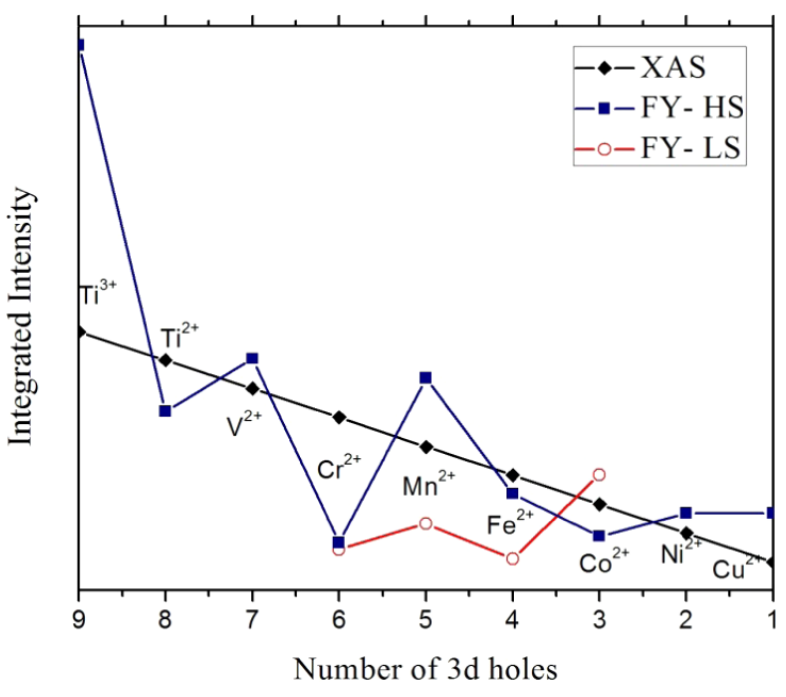

Figure 4. The integrated intensities of the XAS and FY-XAS of $3 d$ metal ions as a function of the number of $3 \mathrm{~d}$ holes. The straight line of the high-spin and low-spin XAS results (black), is compared with high-spin (blue) and low-spin (red) FY-XAS calculations.

\subsection{Low-spin systems}

Figure 3 shows the calculations for the low-spin systems, using a cubic crystal field strength of $10 \mathrm{Dq}=2.4 \mathrm{eV}$. In octahedral symmetry $3 d^{4}, 3 d^{5}, 3 d^{6}$ and $3 d^{7}$ systems contain a high-spin to low-spin transition. The low-spin calculations show a similar trend to the high-spin calculations: (1) an increase of the $\mathrm{L}_{2}$ edge for $\mathrm{Cr}^{2+}, \mathrm{Mn}^{2+}$ and $\mathrm{Fe}^{2+}$ and (2) a transition intensity increase for the states at the ends of the $\mathrm{L}_{2}$ and $\mathrm{L}_{3}$ edges. This is, for example, visible for low-spin $\mathrm{Co}^{2+}$ as a decrease of the FY-XAS intensity at the low energy side of the $\mathrm{L}_{3}$ edge.

\subsection{The consequences for the sum rules}

The intensities of the XAS and FY-XAS spectra as a function of the number of $3 \mathrm{~d}$ holes are plotted in figure 4 . The XAS intensities form a straight line, exactly confirming the $2 p$ XAS sum rule stating that the integrated $2 p$ XAS intensity is proportional to the number of empty $3 d$ states. The FY-XAS shows a fluctuating line due to the difference in the decay channels of FY-XAS. Its relative intensity depends mainly on how many and how strong X-ray emission channels the $2 p$ core states have. This is strongly dependent on the specific symmetry, in other words on the $3 \mathrm{~d}$ count, the crystal field effects and also the charge transfer effects [34, 35]. Figure 4 shows that $3 d^{1}, 3 d^{3}, 3 d^{5}$, low-spin $3 d^{7}, 3 d^{8}$ and $3 d^{9}$ have relatively high FY-XAS integrated intensity, compared with the relatively low FY-XAS intensities of the other $3 \mathrm{~d}$ counts. For example, the $\mathrm{Mn}^{2+}$ system reaches a set of intermediate states that have a larger-than-average integrated $\mathrm{x}$-ray emission decay strength, but it is not evident how the trends in this behavior can be explained. The conclusion is that the sum rule for the number of holes does not apply for FY measurements. This also has implications for the sum rules as used in $\mathrm{X}$-ray magnetic circular dichroism (XMCD). Because

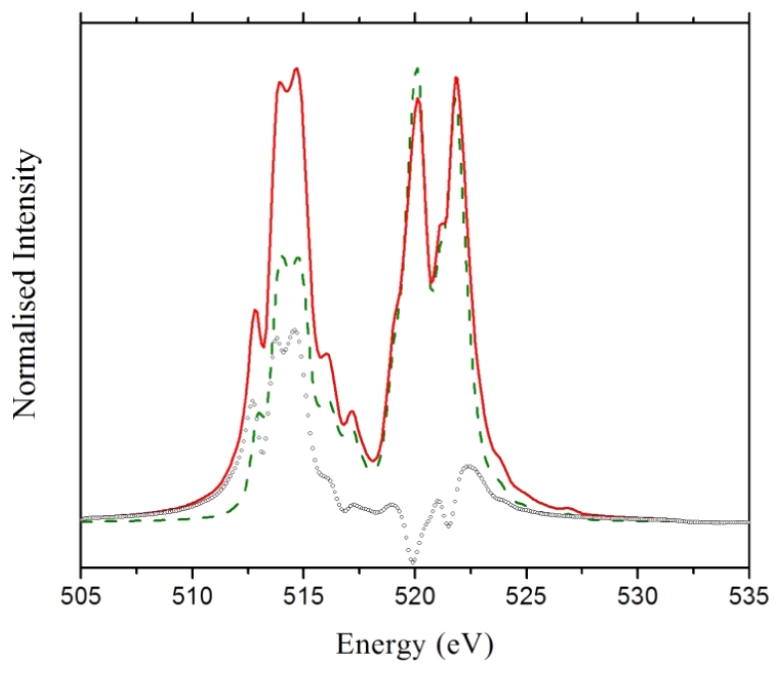

Figure 5. The interference effect on the FY-XAS spectra for $3 \mathrm{~d}^{3}\left(\mathrm{~V}^{2+}\right)$. The solid (red) line shows the spectra with inclusion of the interference effect and the dashed (green) line without it, the difference between the two spectra is shown by the dotted (blue) line.

the FY detected XMCD measurements will also be affected by energy dependent fluorescence decay, systematic deviations will also occur for the XMCD signals. The consequence is that the integrated FY-XAS intensity and the integrated FY-XMCD intensity do not have a well-defined meaning and the XAS and XMCD sum rules do not apply.

\subsection{The effects of interference}

The inclusion of the interference effect can be crucial in some cases as it can create the excitation and decay of intermediate states overlapping in energy. For example, Borgatti et al have shown that in the perpendicular geometry, solely interference effects are responsible for the X-ray magnetic circular dichroism (XMCD) effects in RIXS [32]. A calculation on $3 \mathrm{~d}^{3} \mathrm{~V}^{2+}$ is performed by including the interference effect and plotted along with the FY-XAS omitting the interference effect in figure 5. From the correlation between the two plots, we find that the interference effect is important and is strong at the $\mathrm{L}_{3}$ edge. If interference effects can be neglected one can use a much simpler calculation for the 2p3d RIXS plane, as the Kramers-Heisenberg relation then simplifies to the multiplication of the XAS spectrum with the XES spectrum. What needs to be considered also when interference is neglected is that each $2 \mathrm{p}^{5} 3 \mathrm{~d}^{N+1}$ multiplet state can have a decay channel entirely different from its neighbor state that can be almost degenerate in energy. This implies that also without interference effects one still has to calculate the combination of each specific excitation with its related decay spectrum. A further simplification to the direct multiplication of the XAS spectrum with a fixed XES spectrum is not possible. This is in contrast to systems where multiplet effects are not important and one can use single particle methods such as density functional theory (DFT) to calculate both the XAS and XES spectra [36, 37]. 

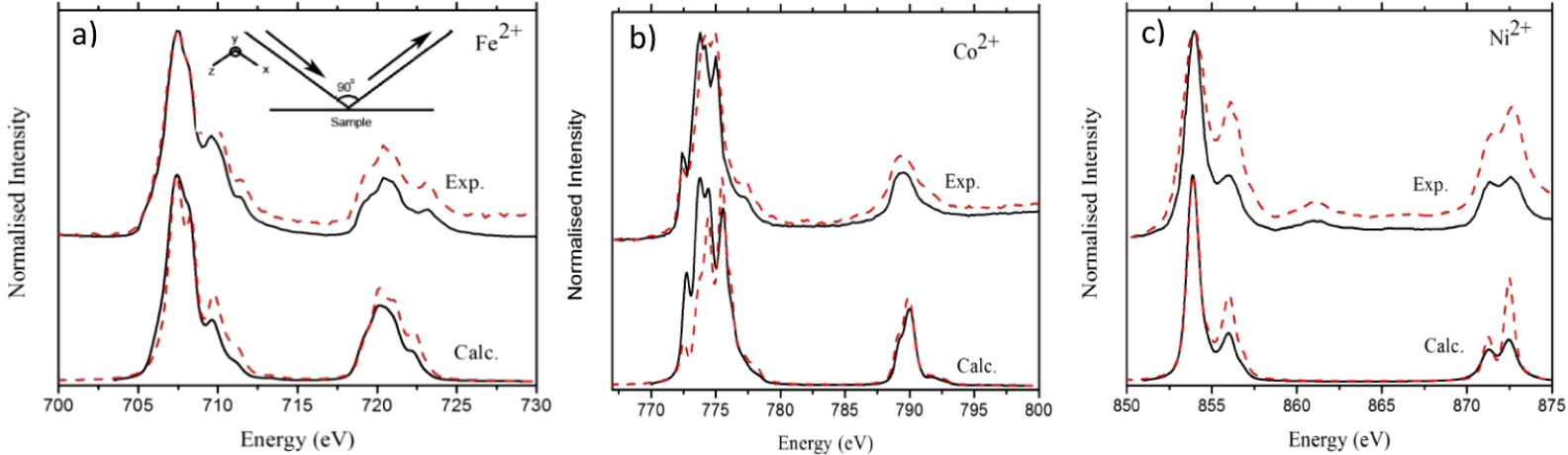

Figure 6. The comparison of the simulated XAS (black, solid line) and FY-XAS (red, dashed line) spectra with the experimental spectra From left to right (a) $\mathrm{Fe}^{2+}$ (measured on $\mathrm{LiFePO}_{4}[24]$ ), (b) $\mathrm{Co}^{2+}$ (measured on $\mathrm{Co}_{2} \mathrm{SiO}_{4}$ [25]) and (c) $\mathrm{Ni}^{2+}$ (experiment on $\left.\mathrm{Cs}\left[\mathrm{NiCr}(\mathrm{CN})_{6}\right] \cdot 2 \mathrm{H}_{2} \mathrm{O}[16]\right)$.

\subsection{Comparison with experiment}

Figure 6 shows the comparison with experiment. We compare the polarization averaged calculations with two bulk oxides $\mathrm{LiFePO}_{4}[27,28]$ and $\mathrm{Co}_{2} \mathrm{SiO}_{4}$ [28] and a coordination compound $\mathrm{Cs}\left[\mathrm{NiCr}(\mathrm{CN})_{6}\right] \cdot 2 \mathrm{H}_{2} \mathrm{O}[16] . \mathrm{LiFePO}_{4}$ contains $3 \mathrm{~d}^{6}$ $\mathrm{Fe}^{2+}, \mathrm{Co}_{2} \mathrm{SiO}_{4}$ contains $3 \mathrm{~d}^{7} \mathrm{Co}^{2+}$ and $\mathrm{Cs}\left[\mathrm{NiCr}(\mathrm{CN})_{6}\right] \cdot 2 \mathrm{H}_{2} \mathrm{O}$ contains $3 \mathrm{~d}^{8} \mathrm{Ni}^{2+}$. For all systems the transmission or electron yield XAS experiments are compared with FY-XAS experiments. The experimental differences between XAS and FY-XAS agree with the calculations. Note that we use the polarization averaged calculations in this figure, which do not agree exactly with the experimental geometries. However, as we showed in figure 2, the difference between the polarization averaged calculations and the LV geometry calculations is not observable with the experimental resolution as available. Figure 6(a) shows the increased $\mathrm{L}_{2}$ edge plus the increased intensity at the high energy side of the $\mathrm{L}_{3}$ edge. Figure 6(b) show the decrease in intensity at the low energy side of the $\mathrm{L}_{3}$ edge and figure 6(c) shows an increase in the intensity at the high energy side of the $\mathrm{L}_{3}$ edge and at the $\mathrm{L}_{2}$ edge. $\mathrm{Cr}^{3+}$ data also confirm the difference between FY-XAS and XAS [19]. All experiments confirm the theoretical predictions. This also implies that for all systems the FY-XAS is dominated by the 2p3d XES decay channel.

\section{Conclusion}

Fluorescence yield measurements of x-ray absorption spectra are often used as a measure of the x-ray absorption cross section. In this paper we have shown that the fluorescence yield decay channels of the $\mathrm{L}_{2,3}$ edges of the transition metals are distinct from the X-ray absorption cross sections, and there is a clear difference between the XAS and FY-XAS spectra in the case of the $3 \mathrm{~d}$ transition metal ions. The results show that FY measurements on transition metal L edges are sensitive to the decay channels and hence cannot be taken as an analog for the electron yield measurements or transmission measurements. This effect is a result of the opening of a fluorescent decay channel for selected absorption transitions. The integrated results show that the sum rule is not applicable on FY-XAS data, which also implies that FY detected XMCD data are affected and cannot be directly used in a quantitative way using the sum rules. The same conclusion can be made regarding the branching ratio. The calculations are confirmed by four experimental data sets comparing FY-XAS and XAS detection, which confirm the theoretical predictions.

\section{References}

[1] Jaklevic J, Kirby J A, Klein M P, Robertson A S, Brown G S and Eisenberger P 1977 Solid State Commun. 23679

[2] Stöhr J 1992 XANES Spectroscopy (Berlin: Springer)

[3] Chen C T, Idzerda Y U, Lin H J, Smith N V, Meigs G, Chaban E, Ho G H, Pellegrin E and Sette F 1995 Phys. Rev. Lett. 75152

[4] de Smit E et al 2008 Nature 456222

[5] Erbil A, Cargill G S III, Frahm R and Boehme R F 1988 Phys. Rev. B 372450

[6] Yang B X and Kirz J 1987 Phys. Rev. B 361361

[7] Schreck S, Gavrila G, Weniger C and Wernet P 2011 Rev. Sci. Instrum. 82103101

[8] Wernet P, Gavrila G, Godehusen K, Weniger C, Nibbering E T J, Elsaesser T and Eberhardt W 2008 Appl. Phys. A 92511

[9] Naslund L A, Luning J, Ufuktepe Y, Ogasawara H, Wernet P, Bergmann U, Pettersson L G M and Nilsson A 2005 J. Phys. Chem. B 10913835

[10] Huse N, Kim T K, Jamula L, McCusker J K, de Groot F M F and Schoenlein R W $2010 \mathrm{~J}$. Am. Chem. Soc. 1326809

[11] Huse N, Wen H D, Nordlund D, Szilagyi E, Daranciang D, Miller T A, Nilsson A, Schoenlein R W and Lindenberg A M 2009 Phys. Chem. Chem. Phys. 113951

[12] Nagasaka M, Hatsui T, Horigome T, Hamamura $Y$ and Kosugi N 2010 J. Electron Spectrosc. 177130

[13] Seidel R et al 2012 J. Am. Chem. Soc. 1341600

[14] de Groot F M F and Kotani A 2008 Core Level Spectroscopy of Solids (Boca Raton, FL: CRC Press)

[15] Nakajima R, Stöhr J and Idzerda Y U 1999 Phys. Rev. B 596421

[16] de Groot F M F, Arrio M A, Sainctavit Ph, Cartier Ch and Chen C T 1994 Solid State Commun. 92991

[17] Pompa M, Flank A M, Lagarde P, Rife J C, Stekhin I, Nakazawa M, Ogasawara H and Kotani A 1997 Phys. Rev. B 562267 
[18] O’Brien W L, Jia J, Dong Q-Y, Callcott T A, Miyano K E, Ederer D L, Mueller D R and Kao C-C 1993 Phys. Rev. Lett. 70238

[19] Wernet P, Kunnus K, Schreck S, Quevedo W, Kurian R, Techert S, de Groot F M F, Odelius M and Föhlisch A 2012 J. Phys. Chem. Lett. submitted

[20] Hocking R K, Wasinger E C, de Groot F M F, Hodgson K O, Hedman B and Solomon E I 2006 J. Am. Chem. Soc. 12810442

[21] Hocking R K, Wasinger E C, Yan Y L, de Groot F M F, Walker F A, Collman J P, Hodgson K O, Hedman B and Solomon E I 2007 J. Am. Chem. Soc. 129113

[22] Thole B T, van der Laan G and Butler P H 1988 Chem. Phys. Lett. 149295

[23] de Groot F M F, Fuggle J C, Thole B T and Sawatzky G A 1990 Phys. Rev. B 425459

[24] Kramers H and Heisenberg W 1925 Z. Phys. A 31681

[25] de Groot F M F 2001 Chem. Rev. 1011779

[26] Ament L J P, van Veenendaal M, Devereaux T P, Hill J P and Brink J v d 2011 Rev. Mod. Phys. 83705

[27] Augustsson A et al 2005 J. Chem. Phys. 123184717
[28] Butorin S M 2012 unpublished

[29] Denecke R, Väterlein P, Bässler M, Wassdahl N, Butorin S, Nilsson A, Rubensson J-E, Nordgren J, Martensson N and Nyholm R 1999 J. Electron Spectrosc. 101-103 971

[30] Ferriani P, Bertoni C M and Ferrari G 2004 Phys. Rev. B 69104433

[31] Borgatti F, Ferriani P, Ghiringhelli G, Tagliaferri A, De Michelis B, Bertoni C M, Brookes N B and Braicovich L 2002 Phys. Rev. B 65094406

[32] Borgatti F, Ghiringhelli G, Ferriani P, Ferrari G, van der Laan G and Bertoni C M 2004 Phys. Rev. B 69134420

[33] Marri I, Bertoni C M, Ferriani P and Joly Y 2006 Phys. Rev. B 74212410

[34] Regier T Z, Achkar A J, Peak D, Tse J S and Hawthorn D G 2012 Nature Chem. 4765

[35] de Groot F M F 2012 Nature Chem. 4766

[36] de Groot F M F, Pizzini S, Fontaine A, Hämäläinen K, Kao C C and Hastings J B 1995 Phys. Rev. B 511045

[37] Smolentsev N, Sikora M, Soldatov A V, Kvasnina K O and Glatzel P 2011 Phys. Rev. B 84235113 